\title{
Microwave-assisted C-C cross-coupling reactions of aryl and heteroaryl halides in water
}

\author{
Kamal M. Dawood* and Moteaa M. El-Deftar \\ Department of Chemistry, Faculty of Science, Cairo University, Giza 12613, Egypt \\ E-mail:
}

\begin{abstract}
The catalytic activity of a benzimidazole-oxime Pd(II)-complex towards Suzuki and Heck C-C cross-coupling reactions of activated and deactivated aryl- and heteroaryl bromides under microwave irradiation as well as thermal heating using water as a green solvent was evaluated. The turnover frequency reached 420,000 under microwave condition.
\end{abstract}

Keywords: Palladium complex, catalysis, microwave, C-C cross-coupling, water solvent

\section{Introduction}

Transition metal-mediated cross-coupling reactions, particularly those based on palladium, have become key transformations in organic synthesis. ${ }^{1}$ Therefore, there has been considerable interest in the development of new, highly-active palladium catalysts that can be used in C-C cross-coupling reactions. In addition, microwave-assisted organic synthesis is advantageous for enabling rapid, reproducible, and scalable chemistry development in research laboratories and industrial processes. ${ }^{2}$ The first microwave-assisted Suzuki cross-coupling reaction was reported in $1996 .{ }^{3}$ Our recent research work is directed to the use of palladium(II) complexes in SuzukiMiyaura and Heck-Mizoroki cross-coupling reactions under microwave heating conditions in aqueous media. ${ }^{4}$ Very recently, we examined the catalytic activity of the $\mathrm{Pd}(\mathrm{II})$-complex $\mathbf{1}$ in cross-coupling reactions of 4-halophenylthiazoles, ${ }^{5}$ herein we report general applications of the Pd(II)-complex 1 as a precatalyst in Suzuki and Heck cross-coupling reactions of aryl- and heteroaryl halides assisted with microwave irradiation using water as a green solvent.

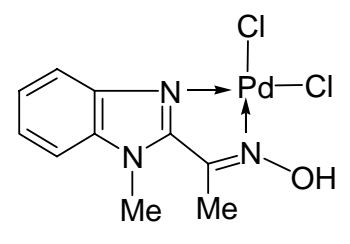




\section{Results and Discussion}

Effect of concentration of the Pd-complex 1 on the Suzuki coupling of 4bromoacetophenone with phenylboronic acid in water under $\mu \mathrm{w}$ irradiation

The effect of concentration of palladium(II) complex 1 on the Suzuki-Miyaura cross-coupling reaction between 4-bromoacetophenone $\mathbf{2 a}$ and phenylboronic acid $\mathbf{3} \mathbf{a}$ in water under microwave irradiation was examined. At first, the reaction was conducted using $1 \mathrm{~mol} \%$ of the precatalyst 1 with $1 \mathrm{mmol}$ 4-bromoacetophenone, $1.2 \mathrm{mmol}$ phenylboronic acid, $0.6 \mathrm{mmol}$ tetrabutylammonium bromide, $2 \mathrm{mmol}$ potassium hydroxide under microwave irradiation (at $160{ }^{\circ} \mathrm{C}, 250$ Watt) in water ( $3 \mathrm{~mL})$ as solvent. TLC showed complete consumption of the starting bromide 2a after 2 min of irradiation. The product 4-acetyl-1,1'-biphenyl (4) was isolated in 97\% yield. Next, the same experiment was repeated using $0.5 \mathrm{~mol} \%$ then with $0.1 \mathrm{~mol} \%$ of the catalyst 1 under identical reaction conditions as above where complete consumption of the bromide 2a was observed after 2 min of irradiation with 93 and 95\% isolated yields, respectively. Furthermore, the same reaction was repeated with $0.05 \mathrm{~mol} \%$ of the precatalyst 1 for $2 \mathrm{~min}$ of $\mu \mathrm{w}$ heating to give full conversion in $96 \%$ isolated yield of compound 4 as shown in Table 1, run 4. Interestingly, when the cross coupling of 2a with 3a was conducted under the same reaction condition using 0.01 and $0.005 \mathrm{~mol} \%$ of complex 1 it resulted in their conversion into compound 4 in 76 and $70 \%$ isolated yields, respectively (Table 1, runs 5 and 6). The high catalytic activity of the Pd-complex 1 can be observed with the very high turnover number (TON) 14000 (TON: the number of mole of product per mole of Pd-complex) and the very high turnover frequency (TOF) $420000 \mathrm{~h}^{-1}$ (TOF: mole of product per mole of Pd-complex per hour) as shown in Table 1, run 6.

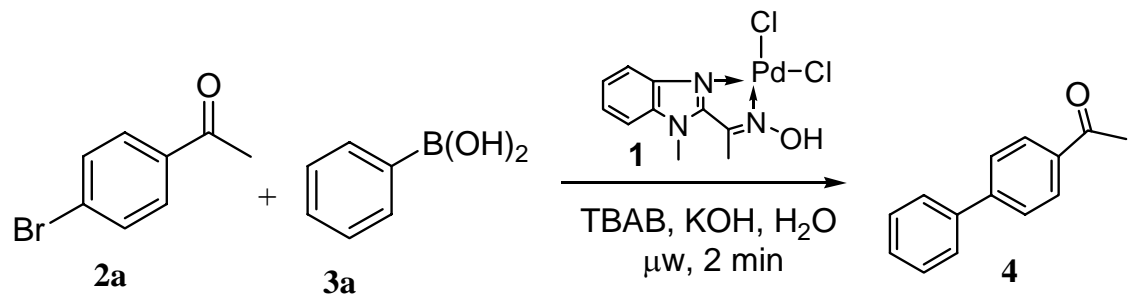


Table 1. Effect of concentration of catalyst 1 on Suzuki coupling of 4-bromoacetophenone with phenylboronic acid in water under microwave heating

\begin{tabular}{lllll}
\hline Entry & mol\% of Pd-Cat. 1 & Yield \% ${ }^{\mathrm{a}, \mathrm{b}}$ & TON & TOF $\left[\mathrm{h}^{-1}\right]$ \\
\hline 1 & 1 & $100(97)$ & 97 & 2910 \\
2 & 0.5 & $100(93)$ & 186 & 5580 \\
3 & 0.1 & $100(95)$ & 950 & 28500 \\
4 & 0.05 & $100(96)$ & 1920 & 57600 \\
5 & 0.01 & $88(76)$ & 7600 & 228000 \\
6 & 0.005 & $86(70)$ & 14000 & 420000 \\
\hline
\end{tabular}

${ }^{a}$ Conditions: 2a $(1.0 \mathrm{mmol}), 3 a(1.2 \mathrm{mmol}), \mathrm{KOH}(2.0 \mathrm{mmol}), \mathrm{TBAB}(0.6 \mathrm{mmol})$, water $(3 \mathrm{~mL})$ at $160{ }^{\circ} \mathrm{C}, 250$ Watt.

${ }^{b}$ Conversion\% based on ${ }^{1} \mathrm{H}$ NMR of the crude reaction product and values between parentheses refer to the isolated yields.

\section{Suzuki-Miyaura cross-coupling of aryl halides with phenylboronic acid using pre-catalyst 1 in water under microwave irradiation}

A general utility of complex $\mathbf{1}$ in Suzuki-Miyaura cross-coupling reactions was investigated as illustrated in Table 2. The Suzuki coupling reactions of the activated aryl bromides 2a-d with phenylboronic acid 3a were carried out under microwave irradiation conditions resulting in the formation of the corresponding biaryl derivatives 4-7 with full conversions in high isolated yields. Under similar reaction conditions as above, the Suzuki coupling between phenylboronic acid 3a with deactivated aryl bromides 2e-g was performed to give excellent yields of the crosscoupled products 8-10 after 2-5 min of microwave heating (Table 2, runs 5-7). 4-Iodobenzoic acid cross-coupled smoothly with phenylboronic acid after one min of microwave heating using $0.5 \mathrm{~mol} \%$ of complex 1 to give $85 \%$ isolated yield of 4-phenylbenzoic acid (6) (Table 2, runs 8).

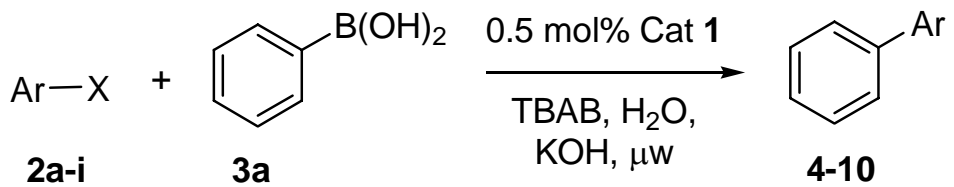


Table 2. Suzuki-Miyaura cross-coupling of aryl halides with phenylboronic acid using precatalyst $\mathbf{1}$ in water under microwave irradiation

Entry

${ }^{a}$ Conditions: Halides 2a-i $(1.0 \mathrm{mmol})$, boronic acid 3a $(1.2 \mathrm{mmol}), \mathrm{KOH}(2.0 \mathrm{mmol}), \mathrm{TBAB}(0.6$ $\mathrm{mmol})$, water $(3 \mathrm{~mL})$ at $160{ }^{\circ} \mathrm{C}, 250$ Watt.

${ }^{b}$ Traces of the starting bromide $2 \mathbf{e}$ were detected by TLC.

${ }^{c}$ The reaction product was treated with few drops of acetic acid.

${ }^{d} 1.0 \mathrm{mmol}$ of TBAB was used. In all runs above, traces of the homo-coupling product (biphenyl) were detected by TLC and mass spectrum. ${ }^{6}$

4-Nitrochlorobenzene $\mathbf{2} \mathbf{i}$, as an example of activated aryl chlorides, was subjected for crosscoupling with phenylboronic acid 3a under microwave heating conditions (Table 2, run 9). Under this condition, the coupled product 5 was obtained in $90 \%$ isolated yield after 3 min of $\mu$ wave irradiation.

As shown in Table 2, the $\operatorname{Pd}(\mathrm{II})$-complex 1 was found to efficiently catalyze the coupling of a wide range of aryl bromides in excellent yields regardless their activating or deactivating substituents. 


\section{Suzuki cross-coupling of aryl(heteroaryl) bromides 2a,j,k with arylboronic acids 3a-d under thermal heating and $\mu \mathrm{w}$ irradiation}

The catalytically active Pd(II)-complex 1 was next applied for Suzuki-Miyaura reaction of aryland heteroaryl bromides $\mathbf{2 a} \mathbf{a}, \mathbf{j}, \mathbf{k}$ with variety of activated and deactivated arylboronic acids 3a-d. Thus, thermal heating of 4-bromoacetophenone 2a with arylboronic acid 3a-d using $0.5 \mathrm{~mol} \%$ of Pd-complex 1 in the presence of $\mathrm{KOH}$ as a base, water as a solvent and TBAB as phase transfer agent for 2 hours gave excellent isolated yields of the corresponding biaryls 4, 11-13 (Table 3 ). Similarly, when the same reactions were repeated under microwave irradiation at $160{ }^{\circ} \mathrm{C}$ and 250 Watt, they gave very high isolated yields of the same biaryls after 2-6 min as shown in Table 3 . Interestingly, the chlorine atom in compound $\mathbf{1 1}$ was survived under both thermal and microwave heating modes and there was no evidence for the formation of 4-(4'chlorobiphenylyl)acetophenone in the crude reaction mixture from the cross coupling of 2a with 4-chlorophenylboronic acid $3 \mathbf{b}$.

Subsequently, the cross-couplings between 4-bromoisoquinoline $2 \mathbf{j}$ and phenylboronic acid 3a was conducted in water using $\mathrm{KOH}$ as a base and TBAB as a phase transfer agent in the presence of $0.5 \mathrm{~mol} \%$ of the $\mathrm{Pd}(\mathrm{II})$-complex 1 to give 4-phenylisoquinoline 14 in $90 \%$ yield after 9 min of microwave irradiation. Three further examples of arylboronic acids $\mathbf{3 b}$-d (activated and deactivated) were similarly allowed to cross-couple with 4-bromoisoquinoline $\mathbf{2 j}$ under the same optimized reaction conditions to afford the corresponding 4-arylisoquinolines $\mathbf{1 5 - 1 7}$ in excellent yields as listed in Table 3, column 3.

Suzuki-Miyaura couplings of 3-bromoquinoline 2k with the arylboronic acids 3a-d were similarly conducted in water under microwave heating conditions using the same molar ratios of the precatalyst $\mathbf{1}$ and the reactants as shown above to give the corresponding 3-arylquinolines 1821 in high yields as illustrated in Table 3, column 4.

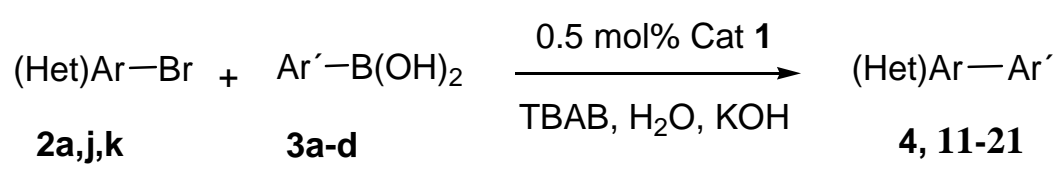


Table 3. Suzuki cross-coupling of aryl (heteroaryl) bromides $\mathbf{2 a , j , k}$ with arylboronic acids 3a-d in water under thermal and microwave heating

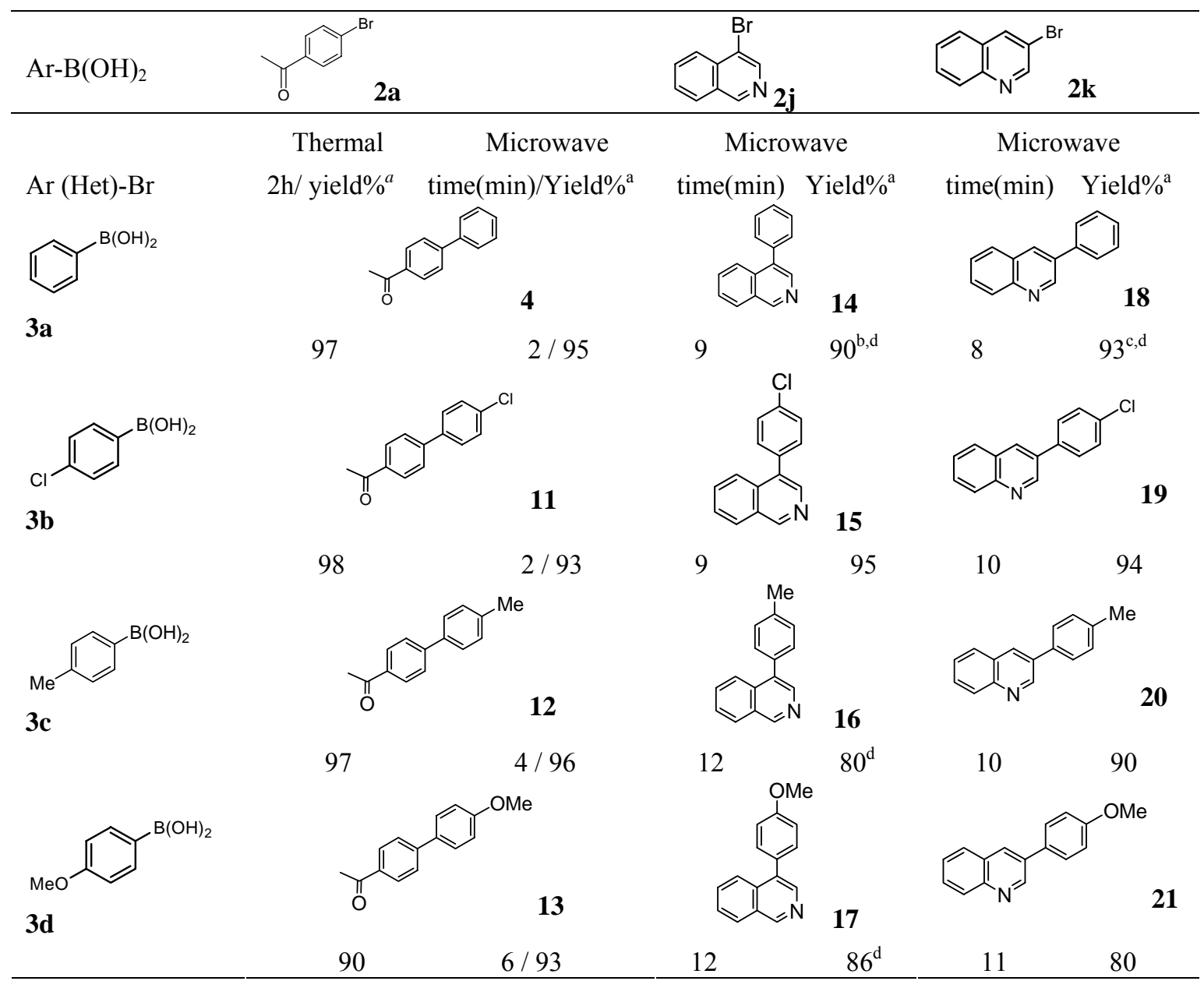

${ }^{\mathrm{a}}$ Conditions: Bromides 2a,j,k $(1.0 \mathrm{mmol})$, boronic acids 3a-d $(1.2 \mathrm{mmol}), \mathrm{KOH}(2.0 \mathrm{mmol})$, TBAB $(0.6 \mathrm{mmol})$, water $(3 \mathrm{~mL})$ at $100{ }^{\circ} \mathrm{C}$ for thermal heating and at $160{ }^{\circ} \mathrm{C}(250$ Watt $)$ for $\mu \mathrm{w}$ irradiation. All values refer to the isolated yields.

${ }^{b}$ Yield was $75 \%$ after 9 min of $\mu \mathrm{w}$ irradiation when catalyst 1 was used in $0.1 \mathrm{~mol} \%$.

${ }^{c}$ Yield was $73 \%$ after 9 min of $\mu \mathrm{w}$ irradiation when catalyst 1 was used in $0.1 \mathrm{~mol} \%$.

${ }^{\mathrm{d}}$ Traces of the starting bromides were detected by TLC.

\section{Mizoroki-Heck cross-coupling of aryl (heteroaryl) bromides in water under microwave irradiation}

The Pd(II) complex-catalyzed cross-coupling reaction of aryl (heteroaryl) bromides $\mathbf{2 a}, \mathbf{j}, \mathbf{k}$ with styrene (Heck reaction) under microwave condition was also investigated. Firstly, the reaction was performed using $0.5 \mathrm{~mol} \%$ of the precatalyst $\mathbf{1}$ for the coupling of 4-bromoacetophenone 2a with styrene 22 in the presence of TBAB and potassium hydroxide in water as solvent under microwave irradiation at $160{ }^{\circ} \mathrm{C}$ for 5 min to give 4-acetylstilbene 23 in $94 \%$ yield (Table 4 ). The same reaction was repeated for 4 -bromoisoquinoline $\mathbf{2} \mathbf{j}$ and 3 -bromoquinoline $\mathbf{2 k}$ with styrene. 
The starting bromides $\mathbf{2} \mathbf{j}, \mathbf{k}$ were completely consumed after $15 \mathrm{~min}$ of microwave irradiation to give the corresponding styryl derivatives 24 and 25 in 86 and $87 \%$ yields, respectively, as outlined in Table 4.

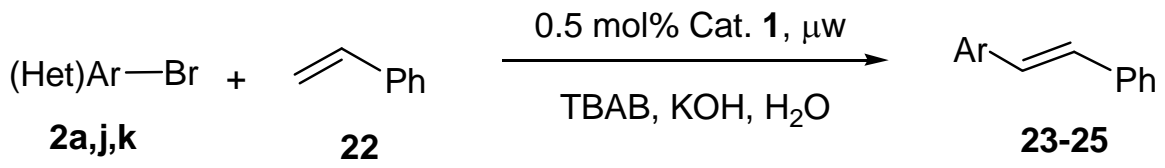

Table 4. Heck cross coupling of aryl (heteroaryl) bromides in water under $\mu \mathrm{w}$ heating

\begin{tabular}{ccccc}
\hline Run & Product & Time (min) & Yield \% \\
\hline 2 & 23 & 5 & 94 \\
\hline
\end{tabular}

${ }^{\mathrm{a} C}$ Conditions: Bromides 2a,j,k $(1 \mathrm{mmol})$; styrene 22 (1.5 mmol); TBAB $(0.6 \mathrm{mmol}) ; \mathrm{KOH}$ ( $2 \mathrm{mmol})$; water $(3 \mathrm{~mL})$, at $160{ }^{\circ} \mathrm{C}(250 \mathrm{Watt})$. All values refer to the isolated yields.

In conclusion, the Pd(II)-complex $\mathbf{1}$ was found to be an efficient and highly active precatalyst for Suzuki-Miyaura and Heck-Miyoroki cross-coupling reactions of activated and deactivated aryl halides as well as heterocyclic bromides. The extremely high turnover number (TON) and turnover frequency (TOF) under microwave irradiation conditions represents the importance of conducting such reactions for mass production on an industrial scale.

\section{Experimental Section}

General. Melting points were determined in open glass capillaries with a Gallenkamp apparatus. The infrared spectra were recorded in potassium bromide disks on a Pye Unicam SP 3-300 and Shimaduz FTIR 8101 PC infrared spectrophotometer. NMR spectra were recorded with a Varian Mercury VXR-300 NMR spectrometer at $300 \mathrm{MHz}\left({ }^{1} \mathrm{H}\right.$ NMR $)$ and at $75 \mathrm{MHz}\left({ }^{13} \mathrm{C}\right.$ NMR) using $\mathrm{CDCl}_{3}$ as solvent and internal standard $\left(\delta 7.27\right.$ and $77.36 \mathrm{ppm}$, for ${ }^{1} \mathrm{H}$ NMR and ${ }^{13} \mathrm{C}$ NMR, respectively). Mass spectra (EI) were obtained at $70 \mathrm{eV}$ with a type Shimadzu GCMQP $1000 \mathrm{EX}$ spectrometer. TON (turnover number) was calculated as: (a catalyst displaying TON 1000 means that the catalyst leads to $100 \%$ conversion of starting materials at a load of $0.1 \% \mathrm{~mol}$ ). Microwave irradiation was carried out using a CEM Discover Labmate ${ }^{\mathrm{TM}}$ microwave apparatus ( $300 \mathrm{~W}$ with ChemDriver ${ }^{\mathrm{TM}}$ Software). Aryl- and heteroaryl halides as well as arylboronic acids were used as purchased without further purification. 
Effect of concentration of Pd(II)-complex 1 on the Suzuki cross-coupling of 4bromoacetophenone with phenylboronic acid in water under microwave irradiation

A mixture of 4-bromoacetophenone 2a (199 mg, $1 \mathrm{mmol})$ and phenylboronic acid 3a (146 mg, $1.2 \mathrm{mmol}$ ), tetrabutylammonium bromide (TBAB) (194 mg, $0.6 \mathrm{mmol}$ ), palladium complex 1 (3.66 mg, $1 \mathrm{~mol} \%), \mathrm{KOH}(112 \mathrm{mg}, 2 \mathrm{mmol})$ and water $(3 \mathrm{~mL})$ was heated under microwave condition at $160{ }^{\circ} \mathrm{C}$ and 250 watt for 2 minutes to give 4-acetyl-1,1'-biphenyl 4. The same experiment was repeated using of palladium precatalyst 1 in different mol\% e.g. $0.5,0.1,0.05$, 0.01 and $0.005 \mathrm{~mol} \%$ with respect to 4-bromoacetophenone. The molar ratio of the reaction components were in all cases as follows; 4-bromoacetophenone, phenylboronic acid, TBAB, $\mathrm{KOH}$, water: $1 / 1.2$ / $0.6 / 2 / 3 \mathrm{~mL}$ water. The product was purified with flash column chromatography using hexane/EtOAc (10:1) as eluent to give 4-acetyl-1,1'-biphenyl 4. The yield $\%$ versus concentration of palladium precatalyst $\mathbf{1}$ is outlined in Table 1.

Suzuki-Miyaura cross-coupling of aryl halides with phenylboronic acid using the precatalyst $\boldsymbol{A}$ in water under microwave irradiation. General procedure

A mixture of the appropriate aryl halide 2a-i $(1 \mathrm{mmol})$, and phenylboronic acid 3a (146 mg, $1.2 \mathrm{mmol}$ ), TBAB (194 mg, $0.6 \mathrm{mmol})$, palladium precatalyst 1 (1.83 $\mathrm{mg}, 0.5 \mathrm{~mol} \%)$ KOH (112 $\mathrm{mg}, 2 \mathrm{mmol})$, and water $(3 \mathrm{~mL})$ were mixed in a glass-process vial. The vial was capped properly, and thereafter the mixture was heated under microwave irradiating conditions at $160{ }^{\circ} \mathrm{C}$ and 250 Watt for the appropriate reaction time as listed in Table 2. After the reaction was almost complete as monitored by TLC, the reaction mixture was extracted with EtOAc $(3 \times 20 \mathrm{~mL})$. The combined organic extracts were dried over anhydrous $\mathrm{MgSO}_{4}$ then filtered and the solvent was evaporated under reduced pressure. The product was purified with flash column chromatography as described above to give the corresponding pure products 4-10.

4-Acetyl-1,1'-biphenyl (4). White solid, mp. $118-120{ }^{\circ} \mathrm{C}$ (Lit. ${ }^{7} \mathrm{mp} .119-120{ }^{\circ} \mathrm{C}$ ); ${ }^{1} \mathrm{H}$ NMR $\left(\mathrm{CDCl}_{3}\right): \delta 2.65\left(\mathrm{~s}, 3 \mathrm{H}, \mathrm{COCH}_{3}\right), 7.46-7.49(\mathrm{~m}, 1 \mathrm{H}), 7.64(\mathrm{~d}, 2 \mathrm{H}, J=7.5 \mathrm{~Hz}), 7.7(\mathrm{~d}, 2 \mathrm{H}, J=8.4$ $\mathrm{Hz}), 7.83$ (d, 2H, $J=8.4 \mathrm{~Hz}), 8.05$ (d, 2H, $J=8.7 \mathrm{~Hz})$; MS m/z (\%) $196\left(\mathrm{M}^{+}, 100\right), 181$ (66.7), 144 (83.3), 87 (90).

4-Nitrobiphenyl (5).Yellow needles; mp. $114-116{ }^{\circ} \mathrm{C}$ (Lit. $\left.{ }^{8} \mathrm{mp} .113-115{ }^{\circ} \mathrm{C}\right) ;{ }^{1} \mathrm{H} \mathrm{NMR}\left(\mathrm{CDCl}_{3}\right)$ $\delta$ 7.46-7.54 (m, 3H), $7.64(\mathrm{~d}, 2 \mathrm{H}, J=9.6 \mathrm{~Hz}), 7.75(\mathrm{~d}, 2 \mathrm{H}, J=9 \mathrm{~Hz}), 8.62(\mathrm{~d}, 2 \mathrm{H}, J=9 \mathrm{~Hz})$; MS $\mathrm{m} / \mathrm{z}(\%) 199$ (M+, 35.6), 169 (28.9), 152 (100), 76 (49.6), 50 (40).

4-Phenylbenzoic acid (6). White crystals; mp. 220-222 ${ }^{\circ} \mathrm{C}$ (Lit. ${ }^{9} \mathrm{mp} .220-221{ }^{\circ} \mathrm{C}$ ); ${ }^{1} \mathrm{H}$ NMR $\left(\mathrm{CDCl}_{3}\right) \delta 7.44-7.54(\mathrm{~m}, 3 \mathrm{H}), 7.66(\mathrm{~d}, 2 \mathrm{H}, J=9.3 \mathrm{~Hz}), 7.72(\mathrm{~d}, 2 \mathrm{H}, J=8.1 \mathrm{~Hz}), 8.21(\mathrm{~d}, 2 \mathrm{H}, J=$ $8.1 \mathrm{~Hz}) ; \mathrm{MS} \mathrm{m} / \mathrm{z}(\%) 198\left(\mathrm{M}^{+}, 100\right), 181$ (44.3), 152 (40.6), 127 (3.8), 76 (5.5).

4-Benzoylbiphenyl (7). Buff crystals, mp. 98-100 ${ }^{\circ} \mathrm{C}$ (Lit. ${ }^{10} \mathrm{mp} .101-102{ }^{\circ} \mathrm{C}$ ); ${ }^{1} \mathrm{H}$ NMR (DMSO$\left.d_{6}\right) \delta 7.41-7.56(\mathrm{~m}, 5 \mathrm{H}), 7.59(\mathrm{~d}, 2 \mathrm{H}, J=7.8 \mathrm{~Hz}), 7.67-7.71(\mathrm{~m}, 1 \mathrm{H}), 7.77(\mathrm{~d}, 2 \mathrm{H}, J=8.1 \mathrm{~Hz})$, $7.83(\mathrm{~d}, 2 \mathrm{H}, J=8.7 \mathrm{~Hz}), 7.87$ (d, 2H, $J=8.7 \mathrm{~Hz})$; MS m/z (\%) $258\left(\mathrm{M}^{+}, 73.5\right), 181$ (100), 152 (51.3), 105 (21), 77 (28). 
1-Phenylnaphthalene (8). Colorless oil; ${ }^{11}{ }^{1} \mathrm{H}$ NMR $\left(\mathrm{CDCl}_{3}\right) \delta 7.65-7.76(\mathrm{~m}, 9 \mathrm{H}), 8.08(\mathrm{~d}, 1 \mathrm{H}, J$ $=7.8 \mathrm{~Hz}), 8.13(\mathrm{~d}, 1 \mathrm{H}, J=7.8 \mathrm{~Hz}), 8.25(\mathrm{~d}, 1 \mathrm{H}, J=8.1 \mathrm{~Hz}) ; \mathrm{MS} \mathrm{m} / \mathrm{z}(\%) 204\left(\mathrm{M}^{+}, 100\right), 189$ (10.1), 176 (12.3), 163 (6.7), 150 (8.1), 101 (42), 88 (10.5).

4-Hydroxybiphenyl (9). White crystals, mp. 166-167 ${ }^{\circ} \mathrm{C}$ (Lit. ${ }^{12} \mathrm{mp} .164-165{ }^{\circ} \mathrm{C}$ ); ${ }^{1} \mathrm{H}$ NMR $\left(\mathrm{CDCl}_{3}\right) \delta 5.05(\mathrm{~s}, 1 \mathrm{H}, \mathrm{OH}), 6.92(\mathrm{~d}, 2 \mathrm{H}, J=7.8 \mathrm{~Hz}), 7.30-7.38(\mathrm{~m}, 1 \mathrm{H}), 7.40-7.45(\mathrm{~m}, 2 \mathrm{H})$, $7.49(\mathrm{~d}, 2 \mathrm{H}, J=8.1 \mathrm{~Hz}), 7.56(\mathrm{~d}, 2 \mathrm{H}, J=8.4 \mathrm{~Hz}) ; \mathrm{MS} \mathrm{m} / \mathrm{z}(\%) 170\left(\mathrm{M}^{+}, 100\right), 141(32.3), 115$ (20.0), 63 (10.3), 51 (12.9).

4-Methoxybiphenyl (10). White solid, mp 87-88 ${ }^{\circ} \mathrm{C}$ (Lit. ${ }^{13} \mathrm{mp} \mathrm{87-88}{ }^{\circ} \mathrm{C}$ ); ${ }^{1} \mathrm{H} \mathrm{NMR}\left(\mathrm{CDCl}_{3}\right) \delta$ $3.87\left(\mathrm{~s}, 3 \mathrm{H}, \mathrm{OCH}_{3}\right), 6.99(\mathrm{~d}, 2 \mathrm{H}, J=8.7 \mathrm{~Hz}), 7.31-7.45(\mathrm{~m}, 3 \mathrm{H}), 7.54(\mathrm{~d}, 2 \mathrm{H}, J=9 \mathrm{~Hz}), 7.57$ (d, $2 \mathrm{H}, J=7.2 \mathrm{~Hz})$; MS m/z (\%) $184\left(\mathrm{M}^{+}, 92.5\right), 169$ (77.5), 141 (70), 115 (55), 91 (52.5), 76 (100), $63(55)$.

Suzuki-Miyaura cross-coupling of 4-bromoacetophenone 2a with arylboronic acids 3a-d in water under thermal heating. General procedure

A mixture of 4-bromoacetophenone 2a (199 $\mathrm{mg}, 1 \mathrm{mmol})$, and the appropriate arylboronic acid 3a-d (1.2 mmol), TBAB (194 mg, $0.6 \mathrm{mmol})$, palladium precatalyst 1 (1.83 $\mathrm{mg}, 0.5 \mathrm{~mol} \%$ ), $\mathrm{KOH}(112 \mathrm{mg}, 2 \mathrm{mmol})$, and water $(3 \mathrm{~mL})$ was shaken at $100{ }^{\circ} \mathrm{C}$ under open air for $2 \mathrm{~h}$ (TLCmonitored) as listed in Table 3. The products were extracted with EtOAc (3 x $20 \mathrm{~mL})$ and the combined organic extracts were dried over anhydrous $\mathrm{MgSO}_{4}$ then filtered and the solvent was evaporated under reduced pressure. The residue was then subjected to separation via flash column chromatography with petroleum ether/EtOAc (10:1) as an eluent to give the corresponding pure products 4 and 11 13, respectively.

Suzuki-Miyaura cross-coupling of aryl (heteroaryl) bromides 2a,j,k with arylboronic acids 3a-d in water under microwave irradiation. General procedure

A mixture of the appropriate bromide $\mathbf{2 a}, \mathbf{2 j}$ or $\mathbf{2 k}$ (199 $\mathrm{mg}, 1 \mathrm{mmol}$ ), and the appropriate arylboronic acid 3a-d (1.2 mmol), TBAB (194 mg, $0.6 \mathrm{mmol})$, palladium complex 1 (1.83 $\mathrm{mg}$, $0.5 \mathrm{~mol} \%), \mathrm{KOH}(112 \mathrm{mg}, 2 \mathrm{mmol})$, and water $(3 \mathrm{~mL})$ were mixed in a process vial. The vial was capped properly and the mixture was then heated under microwave irradiating conditions at $160{ }^{\circ} \mathrm{C}$ and 250 Watt for the appropriate reaction time as listed in Table 3. After the reaction was almost complete (monitored by TLC), the reaction mixture was extracted with EtOAc ( $3 \mathrm{x}$ $20 \mathrm{~mL}$ ) and the combined organic extracts were dried over anhydrous $\mathrm{MgSO}_{4}$ then filtered off and the solvent was evaporated under reduced pressure. The products were purified with flash column chromatography using petroleum ether/EtOAc $(5: 2$ or 10:1) as an eluent to give the corresponding pure cross-coupled products 4 and 11-21 as outlined in Table 3.

4-Chloro-4'-acetyl-1,1'-biphenyl (11). White crystals, mp. 99-100 ${ }^{\circ} \mathrm{C}$ (Lit. ${ }^{14} \mathrm{mp} .98-100{ }^{\circ} \mathrm{C}$ ); ${ }^{1} \mathrm{H} \mathrm{NMR}\left(\mathrm{CDCl}_{3}\right) \delta 2.65\left(\mathrm{~s}, 3 \mathrm{H}, \mathrm{COCH}_{3}\right), 7.45(\mathrm{~d}, 2 \mathrm{H}, J=8.4 \mathrm{~Hz}), 7.56(\mathrm{~d}, 2 \mathrm{H}, J=8.4 \mathrm{~Hz})$, $7.65(\mathrm{~d}, 2 \mathrm{H}, J=8.1 \mathrm{~Hz}), 8.04(\mathrm{~d}, 2 \mathrm{H}, J=8.1 \mathrm{~Hz})$; MS m/z (\%) $232\left(\mathrm{M}^{+}+2,30.4\right), 231\left(\mathrm{M}^{+}+1\right.$, 11.4), $230\left(\mathrm{M}^{+}, 100\right), 151$ (24.3), 114 (16.4), 75 (14.1), 57 (23.2), 50.1 (20.9). 
4-Methyl-4'-acetyl-1,1'-biphenyl (12). White crystals, mp. 122-124 ${ }^{\circ} \mathrm{C}$ (Lit. ${ }^{15} \mathrm{mp} .121-124{ }^{\circ} \mathrm{C}$ ); ${ }^{1} \mathrm{H}$ NMR $\left(\mathrm{CDCl}_{3}\right) \delta 2.42\left(\mathrm{~s}, 3 \mathrm{H}, \mathrm{CH}_{3}\right), 2.64\left(\mathrm{~s}, 3 \mathrm{H}, \mathrm{COCH}_{3}\right), 7.29(\mathrm{~d}, 2 \mathrm{H}, J=7.8 \mathrm{~Hz}), 7.54(\mathrm{~d}$, $2 \mathrm{H}, J=6.6 \mathrm{~Hz}), 7.68(\mathrm{~d}, 2 \mathrm{H}, J=8.7 \mathrm{~Hz}), 8.03(\mathrm{~d}, 2 \mathrm{H}, J=9.6 \mathrm{~Hz}) ; \mathrm{MS} m / z(\%) 210\left(\mathrm{M}^{+}, 49.4\right)$, 195 (100), 165 (22.1), 152 (32.7), 139 (5.3), 115 (5.9), 63 (5.7), 51 (5.5).

4-Methoxy-4'-acetyl-1,1'-biphenyl (13). White crystals, mp. 152-154 ${ }^{\circ} \mathrm{C}$ ( Lit. $^{16} \mathrm{mp} .152-$ $\left.153{ }^{\circ} \mathrm{C}\right) ;{ }^{1} \mathrm{H} \mathrm{NMR}\left(\mathrm{CDCl}_{3}\right) \delta 2.64\left(\mathrm{~s}, 3 \mathrm{H}, \mathrm{COCH}_{3}\right), 3.88\left(\mathrm{~s}, 3 \mathrm{H}, \mathrm{OCH}_{3}\right), 7.01(\mathrm{~d}, 2 \mathrm{H}, \mathrm{J}=9 \mathrm{~Hz})$, $7.59(\mathrm{~d}, 2 \mathrm{H}, J=8.7 \mathrm{~Hz}), 7.66(\mathrm{~d}, 2 \mathrm{H}, J=8.7 \mathrm{~Hz}), 8.02(\mathrm{~d}, 2 \mathrm{H}, J=8.7 \mathrm{~Hz})$; MS m/z (\%) $226\left(\mathrm{M}^{+}\right.$, 57.9), 211 (100), 183 (18.5), 168 (23), 152 (12.4), 139 (36.3), 105 (5.7), 63 (8.4).

4-Phenylisoquinoline (14). Yellow oil; ${ }^{17}{ }^{1} \mathrm{H} \mathrm{NMR}\left(\mathrm{CDCl}_{3}\right) \delta$ 7.48-7.64 (m, 7H), 7.89-8.02 (m, 2H), 8.48 (s, 1H), 9.24 (s, 1H); MS m/z (\%) 205 ( $\left.\mathrm{M}^{+}, 100\right), 176$ (25.4), 148 (26.9), 102 (25), 88 (17), 77 (27.9).

4-(4-Chlorophenyl)isoquinoline (15). White crystals, mp. 84-86 ${ }^{\circ} \mathrm{C} ;{ }^{1} \mathrm{H} \mathrm{NMR}\left(\mathrm{CDCl}_{3}\right) \delta 7.45$ $7.54(\mathrm{~m}, 4 \mathrm{H}), 7.63-7.72(\mathrm{~m}, 2 \mathrm{H}), 7.87(\mathrm{~d}, 1 \mathrm{H}, J=8.1 \mathrm{~Hz}), 8.06(\mathrm{~d}, 1 \mathrm{H}, J=8.7 \mathrm{~Hz}), 8.47(\mathrm{~s}, 1 \mathrm{H})$, $9.28(\mathrm{~s}, 1 \mathrm{H}) ;{ }^{13} \mathrm{C} \mathrm{NMR}\left(\mathrm{CDCl}_{3}\right) \delta 124.4,127.6,128,128.8,130.8,131.3,134,134.2,135.3$, 142.5, 152.1; MS m/z (\%) $241\left(\mathrm{M}^{+}+2,34.7\right), 240\left(\mathrm{M}^{+}+1,20\right), 239\left(\mathrm{M}^{+}, 100\right), 204$ (54.2), 176 (28.9), 101 (21.4), 88 (33.3), 75 (16.9). Anal. Calcd for $\mathrm{C}_{15} \mathrm{H}_{10} \mathrm{ClN}$ : C, 75.16; H, 4.21; N, 5.84\%. Found: C, 75.12; H, 4.31; N, 5.98\%.

4-(4-Methylphenyl)isoquinoline (16). Colorless oil; ${ }^{18}{ }^{1} \mathrm{H} \mathrm{NMR}\left(\mathrm{CDCl}_{3}\right) \delta 2.47\left(\mathrm{~s}, 3 \mathrm{H}, \mathrm{CH}_{3}\right)$, $7.34(\mathrm{~d}, 2 \mathrm{H}, J=7.8 \mathrm{~Hz}), 7.42(\mathrm{~d}, 2 \mathrm{H}, J=6.9 \mathrm{~Hz}), 7.58-7.67(\mathrm{~m}, 2 \mathrm{H}),, 7.94(\mathrm{~d}, 1 \mathrm{H}, J=7.8 \mathrm{~Hz})$, $8.02(\mathrm{~d}, 1 \mathrm{H}, J=8.1 \mathrm{~Hz}), 8.50(\mathrm{~s}, 1 \mathrm{H}), 9.25(\mathrm{~s}, 1 \mathrm{H}) ;{ }^{13} \mathrm{C} \mathrm{NMR}\left(\mathrm{CDCl}_{3}\right) \delta 21.2,124.8,127.0$, $127.8,128.4,129.3,129.9,130.4,133.2,134.0,134.3,137.7,142.8,151.7$.

4-(4-Methoxyphenyl)isoquinoline (17). White crystal, mp. 82-84 ${ }^{\circ} \mathrm{C}$ (lit. ${ }^{18} \mathrm{mp} .82-84{ }^{\circ} \mathrm{C}$ ); ${ }^{1} \mathrm{H}$ $\operatorname{NMR}\left(\mathrm{CDCl}_{3}\right) \delta 3.91\left(\mathrm{~s}, 3 \mathrm{H}, \mathrm{OCH}_{3}\right), 7.07(\mathrm{~d}, 2 \mathrm{H}, J=8.1 \mathrm{~Hz}), 7.46(\mathrm{~d}, 2 \mathrm{H}, J=7.8 \mathrm{~Hz}), 7.60-7.7$ $(\mathrm{m}, 2 \mathrm{H}), 7.95(\mathrm{~d}, 1 \mathrm{H}, J=8.1 \mathrm{~Hz}), 8.04(\mathrm{~d}, 1 \mathrm{H}, J=7.5 \mathrm{~Hz}), 8.49(\mathrm{~s}, 1 \mathrm{H}), 9.25(\mathrm{~s}, 1 \mathrm{H}) ; \mathrm{MS} \mathrm{m} / \mathrm{z}$ (\%) $235\left(\mathrm{M}^{+}, 100\right), 220$ (60.2), 191 (39.1), 165 (40.7), 95 (8.9), 88 (8.7), 63 (9.1).

3-Phenylquinoline (18). Pale yellow powder, mp. 50-51 ${ }^{\circ} \mathrm{C}$ (lit. $\left.{ }^{19} \mathrm{mp} .52{ }^{\circ} \mathrm{C}\right) ;{ }^{1} \mathrm{H} \mathrm{NMR}\left(\mathrm{CDCl}_{3}\right)$ $\delta$ 7.39-7.45 (m, 4H), 7.48-7.70 (m, 3H), $7.80(\mathrm{~d}, 1 \mathrm{H}, J=8.1 \mathrm{~Hz}), 8.16(\mathrm{~d}, 1 \mathrm{H}, J=8.7 \mathrm{~Hz}), 8.21$ (s, 1H), 9.18 (s, 1H); MS m/z (\%) $205\left(\mathrm{M}^{+}, 100\right), 176$ (14.4), 151 (7.8), 102 (14.1), 88 (16.5), 76 (38.4), 63 (16.3).

3-(4-Chlorophenyl)quinoline (19). White crystals, mp. $134-136{ }^{\circ} \mathrm{C}$ (Ref. $\left.{ }^{20} \mathrm{mp} .133-134{ }^{\circ} \mathrm{C}\right) ;{ }^{1} \mathrm{H}$ $\operatorname{NMR}\left(\mathrm{CDCl}_{3}\right) \delta 7.50(\mathrm{~d}, 2 \mathrm{H}, J=8.7 \mathrm{~Hz}), 7.58-7.66(\mathrm{~m}, 3 \mathrm{H}), 7.73-7.78(\mathrm{~m}, 1 \mathrm{H}), 7.90(\mathrm{~d}, 1 \mathrm{H}, J=$ $8.1 \mathrm{~Hz}), 8.15(\mathrm{~d}, 1 \mathrm{H}, J=8.4 \mathrm{~Hz}), 8.28(\mathrm{~d}, 1 \mathrm{H}, J=1.8 \mathrm{~Hz}), 9.15(\mathrm{~d}, 1 \mathrm{H}, J=2.1 \mathrm{~Hz}) ; \mathrm{MS} \mathrm{m} / \mathrm{z}(\%)$ $241\left(\mathrm{M}^{+}+2,37.1\right), 240\left(\mathrm{M}^{+}+1,26.6\right), 239\left(\mathrm{M}^{+}, 100\right), 204$ (37.3), 176 (25.3), 102 (12.2), 88 (45.7), 75 (29.6).

3-(4-Methylphenyl)quinoline (20). White crystal, mp. $72-74{ }^{\circ} \mathrm{C}\left(1 \mathrm{it} .{ }^{21} \mathrm{mp} 72-73{ }^{\circ} \mathrm{C}\right) ;{ }^{1} \mathrm{H} \mathrm{NMR}$ $\left(\mathrm{CDCl}_{3}\right) \delta 2.45\left(\mathrm{~s}, 3 \mathrm{H}, \mathrm{CH}_{3}\right), 7.35(\mathrm{~d}, 2 \mathrm{H}, J=8.4 \mathrm{~Hz}), 7.57-7.67(\mathrm{~m}, 3 \mathrm{H}), 7.72-7.77(\mathrm{~m}, 1 \mathrm{H})$, $7.88(\mathrm{~d}, 1 \mathrm{H}, J=8.1 \mathrm{~Hz}), 8.15(\mathrm{~d}, 1 \mathrm{H}, J=8.4 \mathrm{~Hz}), 8.29(\mathrm{~s}, 1 \mathrm{H}), 9.2(\mathrm{~s}, 1 \mathrm{H}) ; \mathrm{MS} \mathrm{m} / \mathrm{z}(\%) 219\left(\mathrm{M}^{+}\right.$, 100), 189 (7.8), 110 (7.6), 109 (11.8), 108 (11.2), 95 (17.4), 94 (8.1).

3-(4-Methoxyphenyl)quinoline (21). White crystals, mp. 84-86 ${ }^{\circ} \mathrm{C}$ (Lit. ${ }^{22} \mathrm{mp} .83-85{ }^{\circ} \mathrm{C}$ ); ${ }^{1} \mathrm{H}$ $\operatorname{NMR}\left(\mathrm{CDCl}_{3}\right) \delta 3.89\left(\mathrm{~s}, 3 \mathrm{H}, \mathrm{OCH}_{3}\right), 7.07(\mathrm{~d}, 2 \mathrm{H}, J=9 \mathrm{~Hz}), 7.54-7.74(\mathrm{~m}, 4 \mathrm{H}), 7.86(\mathrm{~d}, 1 \mathrm{H}, J=$ 
$8.4 \mathrm{~Hz}), 8.14(\mathrm{~d}, 1 \mathrm{H}, J=8.4 \mathrm{~Hz}), 8.25(\mathrm{~s}, 1 \mathrm{H}), 9.18(\mathrm{~s}, 1 \mathrm{H}) ; \mathrm{MS}$ m/z (\%) $235\left(\mathrm{M}^{+}, 100\right), 220$ (32.5), 204 (13), 192 (56.3), 177 (15.2), 163 (25.1), 153 (14.7), 132 (13), 117 (19.9), 101 (11.7), 95 (16), 85 (12.1), 76 (12.6), 63 (19).

Mizoroki-Heck cross-coupling of styrene with aryl (heteroaryl) bromides 2a,j,k in water under microwave heating

A mixture of the appropriate aryl or heteroaryl bromide $\mathbf{2 a , j , k}(1 \mathrm{mmol})$ and styrene 22 (1.5 mmol), TBAB (0.6 mmol), precatalyst 1 (1.83 $\mathrm{mg}, 0.5 \mathrm{~mol} \%)$, and potassium hydroxide $(112 \mathrm{mg}, 2 \mathrm{mmol})$ in water $(3 \mathrm{~mL})$ was mixed in a process vial. The vial was capped properly, and the mixture was then heated under microwave irradiating conditions at $150{ }^{\circ} \mathrm{C}$ and 250 Watt for the appropriate reaction time as listed in Table 4. After the reaction was complete as monitored by TLC, the reaction mixture was left to cool to room temperature. The reaction mixture was then extracted three times with EtOAc $(3 \times 20 \mathrm{~mL})$ and then the organic fractions were combined together, dried over $\mathrm{MgSO}_{4}$, filtered and then the solvent was removed under vacuum. The residue was then subjected to purification via flash column chromatography with petroleum ether-EtOAc (10:1) as eluent to give the corresponding pure products 23 25.

(E) 4-Acetylstilbene (23). Colorless crystals, mp 143-144 ${ }^{\circ} \mathrm{C}$ (Lit. ${ }^{21,22} \mathrm{mp}$. 141-142 ${ }^{\circ} \mathrm{C}$ ); ${ }^{1} \mathrm{H}$ NMR $\left(\mathrm{CDCl}_{3}\right) \delta 2.62\left(\mathrm{~s}, 3 \mathrm{H}, \mathrm{COCH}_{3}\right), 7.14(\mathrm{~d}, 1 \mathrm{H}, J=16.5 \mathrm{~Hz}), 7.25(\mathrm{~d}, 1 \mathrm{H}, J=15.3 \mathrm{~Hz}), 7.31-$ $7.42(\mathrm{~m}, 3 \mathrm{H}), 7.54-7.61(\mathrm{~m}, 4 \mathrm{H}), 7.97(\mathrm{~d}, 2 \mathrm{H}, \quad J=8.4 \mathrm{~Hz}) ;{ }^{13} \mathrm{C} \mathrm{NMR}\left(\mathrm{CDCl}_{3}\right) \delta 26.9,126.8$, 127.1, 127.7, 128.6, 129.1, 129.2, 131.7, 136.2, 136.9, 142.3, 197.7; MS m/z (\%) $222\left(\mathrm{M}^{+}, 57.2\right)$, 207 (100), 178 (67.2), 152 (10), 104 (11.6), 89 (42.2), 76 (27.7), 63 (14.9), 51 (25.6).

(E) 4-(Styryl)isoquinoline (24). Pale yellow powder, mp. $73-74{ }^{\circ} \mathrm{C}\left(\mathrm{Lit}^{23} \mathrm{mp} .75{ }^{\circ} \mathrm{C}\right) ;{ }^{1} \mathrm{H} \mathrm{NMR}$ $\left(\mathrm{CDCl}_{3}\right) \delta 7.13(\mathrm{~d}, 1 \mathrm{H}, J=16.2 \mathrm{~Hz}), 7.30-7.41(\mathrm{~m}, 3 \mathrm{H}), 7.52-7.58(\mathrm{~m}, 4 \mathrm{H}), 7.66(\mathrm{~d}, 1 \mathrm{H}, J=14.7$ $\mathrm{Hz}), 7.89(\mathrm{~d}, 1 \mathrm{H}, J=8.1 \mathrm{~Hz}), 8.06(\mathrm{~d}, 1 \mathrm{H}, J=8.7 \mathrm{~Hz}), 8.74(\mathrm{~s}, 1 \mathrm{H}), 9.13(\mathrm{~s}, 1 \mathrm{H}) ;{ }^{13} \mathrm{C}$ NMR $\left(\mathrm{CDCl}_{3}\right) \delta 122.8,123.3,127.2,127.5,128.4,128.5,128.6,128.9,129.1,130.8,133.5,134.1$, 137.4, 140.8, 152.2; MS m/z (\%) $231\left(\mathrm{M}^{+}, 37\right), 230$ (100), 202 (15.1), 128 (3), 115 (7.8), 101 (10.4), 88 (7), 76 (7.2).

(E) 3-(Styryl)quinoline (25). Yellowish-white crystals, mp. 97-98 ${ }^{\circ} \mathrm{C}$ (Lit. ${ }^{16} \mathrm{mp} .98-99{ }^{\circ} \mathrm{C}$ ); ${ }^{1} \mathrm{H}$ $\operatorname{NMR}\left(\mathrm{CDCl}_{3}\right) \delta 7.24(\mathrm{~d}, 1 \mathrm{H}, J=16.5 \mathrm{~Hz}), 7.19-7.44(\mathrm{~m}, 4 \mathrm{H}), 7.56-7.60(\mathrm{~m}, 3 \mathrm{H}), 7.69(\mathrm{~d}, 1 \mathrm{H}, J$ $=15.3 \mathrm{~Hz}), 7.83(\mathrm{~d}, 1 \mathrm{H}, J=8.1 \mathrm{~Hz}), 8.12(\mathrm{~d}, 1 \mathrm{H}, J=8.4 \mathrm{~Hz}), 8.19(\mathrm{~s}, 1 \mathrm{H}), 9.14(\mathrm{~s}, 1 \mathrm{H}) ;{ }^{13} \mathrm{C}$ NMR $\left(\mathrm{CDCl}_{3}\right) \delta 125.5,127.0,127.3,128.1,128.4,128.6,129.1,129.5,129.6,130.6,131.2$, 132.6, 137.1, 147.8, 149.8; MS m/z (\%) $231\left(\mathrm{M}^{+}, 73.2\right), 230$ (100), 202 (16.1), 115 (20.4), 101 (24.1), 76 (15.2), 63 (14.3), 51 (13.4).

\section{References}

1. (a) Handbook of Organopalladium Chemistry for Organic Synthesis; Negishi, E., Ed.; WileyInterscience: Hoboken, USA, 2002. (b) Miyaura, N. J. Organomet. Chem., 2002, 653, 54. (c) Suzuki, A. J. Organomet. Chem., 2002, 653, 83. (d) Dounay, A. B.; Overman, L. E. Chem. 
Rev. 2003, 103, 2945. (e) Bellina, F.; Carpita, A.; Rossi, R. Synthesis 2004, 15, 2419. (f) Alonso, F.; Beletskaya, I. P.; Yus, M. Tetrahedron 2008, 64, 3047.

2. Reviews on microwave assisted synthesis: (a) Cablewski, T.; Faux, A. F.; Strauss, C. R. J. Org. Chem. 1994, 59, 3408. (b) Microwaves in Organic Synthesis, Loupy, A. Ed., WileyVCH, 2002. (c) Kappe, C. O. Curr. Opin. Chem. Biol. 2002, 6, 314. (d) Farina, V. Adv. Synth. Catal. 2004, 346, 1553. (e) Desai, B.; Kappe, C. O. In Immobilized Catalysts Kirschning, A., Ed. Top. Curr. Chem. 2004, 242, 177. (f) Kappe, C. O. Angew. Chem. Int. Ed. 2004, 43, 6250. (g) Hoz, A.; Ortiz, A.D.; Moreno, A. Chem. Soc. Rev. 2005, 34, 164. (h) Leadbeater, N. E. Chem. Commun. 2005, 2881. (i) Kappe, C. O.; Dallinger, D. Nat. Rev. Drug. Discovery 2006, 5, 55.

3. (a) Larhed, M.; Hallberg, A. J. Org. Chem., 1996, 61, 9582. (b) Larhed, M.; Lindeberg, G.; Hallberg, A. Tetrahedron Lett. 1996, 37, 8219.

4. (a) Dawood, K. M.; Kirschning, A. Tetrahedron 2005, 61, 12121. (b) Solodenko, W.; Dawood, K. M.; Brochwitz, C.; Wartchow, R.; Hashem, Md. A.; Vaultier, M.; Kirschning, A. Mol. Div. 2005, 9, 333. (c) Dawood, K. M.; Solodenko, W.; Kirschning, A. Arkivoc 2007, (v), 104. (d) Dawood, K. M. Tetrahedron, 2007, 63, 9642. (e) Dawood, K. M.; Fayed, M. S.; Elkhalea, M. M. Arkivoc 2009, (xiii), 324.

5. Dawood, K. M.; El-Deftar, M. M. Synthesis 2010, 1030.

6. (a) Miyaura, N.; Suzuki, A. Main Group Met. Chem. 1987, 10, 295. (b) Wong, M. S.; Zhang, X. L. Tetrahedron Lett. 2001, 42, 4087.

7. Zhu, L.; Duquette, J.; Zhang, M. J. Org. Chem. 2003, 68, 3729.

8. Wallow, T. L.; Novak, B. M. J. Org. Chem. 1994, 59, 5034.

9. Huang, Y.-T; Wang, Y.-H. Acta Chimica Sinica 1954, 20, 10.

10. (a) Sapountzis, I. Angew. Chem. Int. Ed. 2005, 44, 1654. (b) Rao, M. L. N. Tetrahedron 2008, 64, 5762.

11. Shelby, N.Q.; Stambuli, J. P.; Hartwig, J. F. J. Org. Chem. 2002, 67, 5553.

12. Minabe, M. J. Org. Chem. 1987, 52, 1745.

13. Zhang, G. J. Chem. Res. 2004, 9, 593.

14. Musante, C.; Parrini, V. Gazz. Chim. Ital. 1949, 79, 453.

15. Dovgosheya, M. I.; Mil'ner, R. S. Zh. Obshch. Khim. 1964, 34, 977.

16. Denmark, S. E.; Ober, M. H. Org. Lett. 2003, 5, 1357.

17. Becht, J. M.; Gissot, A.; Wagner, A.; Mioskowski, C. Chem. Eur. J. 2003, 9, 3209.

18. Ishikura, M.; Oda, I.; Terashima, M. Heterocycles 1987, 26, 1603.

19. (a) Rudler, H.; Reville, T. D. J. Organomet. Chem. 2001, 617-618, 571. (b) Ishikura, M.; Oda, I.; Terashima, M. Heterocycles 1985, 23, 2375.

20. Doi, H.; Sakai, T.; Iguchi, M.; Yamada, K.-I.; Tomioka, K. J. Am. Chem. Soc. 2003, 125, 2886.

21. Cacchi, S.; Fabrizi, G.; Marinellli, F.; Moro, L.; Pace, P. Tetrahedron 1996, 52, 10225.

22. Bezou, P.; Hilberer, A.; Hadziioannou, G. Synthesis 1996, 449.

23. Loader, C. E.; Timmons, C. J. J. Chem. Soc. 1968, 330. 